\title{
Experimental Confirmation Of FK Concentrator Insensitivity To Chromatic Aberrations
}

\author{
P. Zamora , P. Espinet-González , P. Benitez , I. García , I. Rey-Stolle , J.C. \\ Miñano , C. Algora
}

\begin{abstract}
The FK concentrator has demonstrated during the last years that compares very well with other Fresnel-based concentrator optics for CPV. There are several features that provide the FK high performance: (1) high optical efficiency; (2) large tolerance to tracking misalignment and manufacturing errors, thanks to a high CAP (ConcentrationAcceptance Product); (3) good irradiance uniformity and low chromatic dispersion on the cell surface. Non-uniformities in terms of absolute irradiance and spectral content produced by conventional CPV systems can originate electrical losses in multi-junction (MJ) solar cells. The aim of this work is to analyze the influence of these non-uniformities in the FK concentrator performance and how FK concentrator provides high electrical efficiencies thanks to its insensitivity to chromatic aberrations, especially when components move away from the module nominal position due to manufacturing misalignments. This analysis has been done here by means of both, experimental on-sun measurements and simulations based on 3D fully distributed circuit model for MJ cells.
\end{abstract}

Keywords: Fresnel lens, nonimaging, solar concentrator, Köhler integration, CPV, multi-junction solar cells PACS: 42.15.Eq, 42.79.Ek, 88.40.F, 88.40.H, 88.40.jj, 88.40.fc, 88.40.jp, 81.05.Ea

\section{INTRODUCTION}

Conventional CPV optical concentrators provide nonideal irradiance profiles on the cell surface, presenting certain degree of non-uniformity and chromatic dispersion. Irradiances with uneven profiles and high chromatic dispersion lead to losses in $F F$ (Fill-Factor) and electrical efficiency, seriously affecting the whole module performance. Additionally, when the module presents manufacturing or operational misalignments (i.e. some module components are not at their nominal positions, as happens in module real operation), these negative effects are magnified entailing even larger module efficiency losses. The FK (Fresnel-Köhler) [1], a concentrator providing even irradiance and low chromatic dispersion profiles even when the module is not perfectly aligned, ensures high electrical efficiency in large-scale CPV arrays thanks to its insensitivity to misalignments.

This FK insensitivity in terms of electrical efficiency is demonstrated here by means of modifying its POE-cell distance (being POE the concentrator's Primary Optical Element) around its nominal value, and showing both, simulations and experimental measurements. The simulations combine two enhanced modeling tools, namely, ray-tracing and 3D distributed models. The irradiance on each subcell provided by the concentrator is obtained by ray-tracing simulations and it is then used to calculate the actual power delivered by the cell. The IV curves of the MJ cell inside the concentrator are obtained by using an advanced 3D distributed model [2] for triple-junction solar cells already described in [3].

Results will show that both $F F$ and cell efficiency maintain rather constant values along wide ranges of POE-cell distance, proving the insensitivity to chromatic aberrations of the FK concentrator.

\section{MODEL}

The simulations must reproduce faithfully the conditions of the outdoor measurements carried out on our FK concentrator prototype in order to compare both in a fair way. This implies that the spectral characteristics of the simulated sunlight must be identical to that during the measurement. Solar spectrum has been generated using NREL's SMARTS software, in this particular case for a July day in Madrid (Spain) at $4 \mathrm{pm}$. A moment close to solar midday has been chosen in order to have both very 
stable solar irradiance and spectral characteristics during the whole measurement. Note that for this solar spectrum $J_{\text {ratio }}=J_{\text {top }} / J_{\text {mid }}=1.055$ which means that the solar cell is slightly away from current matching situation. $J_{\text {ratio }}$ values closer to 1 would produce higher $I_{s c}$ and efficiency, this is why results shown below do not correspond to FK peak efficiencies (over $32 \%$, as presented in [4]).

The optical model for the concentrator and the electrical model for the solar cell are briefly described in the next sections. A more detailed description of the simulation model (optics and cell) can be found in previous works $[2,3,5]$.

\section{Optical Model}

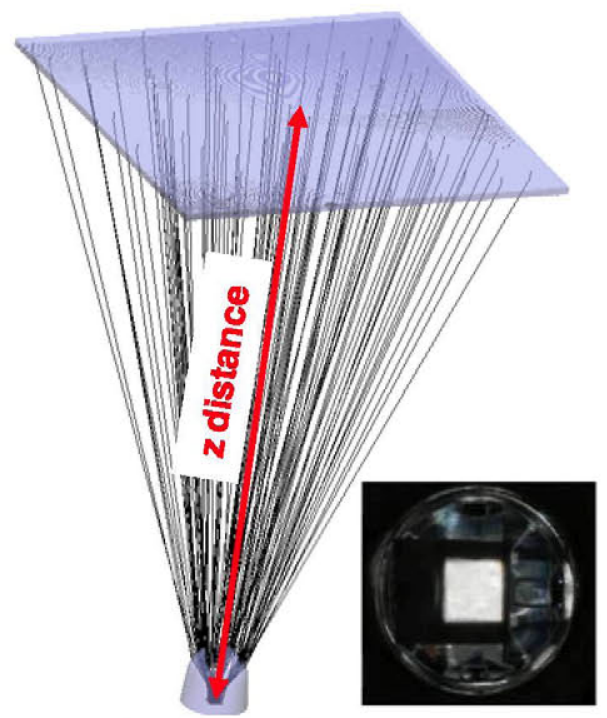

FIGURE 1 Rendered view of FK concentrator during a raytrace simulation, with $\mathrm{z}$ axis marked in red color. At bottom right a real image of the excellent irradiance on the cell achieved by the FK concentrator

The optical performance of the concentrator is modeled through ray tracing. The FK concentrator analyzed for this work presents the next features: geometrical concentration over cell illuminated area $C_{g}=1,080 \mathrm{X} ; f / 1.05$; POE-cell distance $z=250 \mathrm{~mm}$ (at nominal position); POE made of PMMA with $2^{\circ}$ draft angles; SOE (Secondary Optical Element) made of B270 glass (without AR coating). Fresnel losses, refractive index and absorption are considered as a function of wavelength in all simulations. No temperature effects have been considered in the optical model, so small variations in POE foci positions have not been taken into account.

FIGURE 1 shows a 3D render image of the FK concentrator analyzed, for which POE-cell distance (marked in red color) will be modified in order to study the module performance dependence on it. Note that an FK concentrator is a 4-fold architecture where rays refracted by one fold of the POE must hit the corresponding SOE fold (as shown in the figure).

\section{Solar Cell Model}

The solar cell model used here is based on dividing the solar cell into elementary units and assigning a suitable circuit model to each unit, depending on its geometry and position in the solar cell area (external contact, metalized, illuminated, or perimeter). The complete solar cell can be subsequently modeled by an electrical circuit that is obtained by interconnecting every unitcircuit with its neighbors. This solar cell model allows that simulated performance of the system will not just depend on the total amount of light reaching the cell and its spectral characteristics, but also on its particular distribution impinging the cell.

The solar cell simulated is a triple-junction cell with an active area of $5.5 \mathrm{~mm} \times 5.5 \mathrm{~mm}$. The top subcell has a $J_{s c}$ of $12.5 \mathrm{~mA} / \mathrm{cm}^{2}$, the middle subcell, a $J_{s c}$ of $12.7 \mathrm{~mA} / \mathrm{cm}^{2}$ and the bottom subcell, a $J_{s c}$ of 19.3 $\mathrm{mA} / \mathrm{cm}^{2}$ (all of them at 1 sun $\left(900 \mathrm{~W} / \mathrm{m}^{2}\right)$. Cell $J_{s c}$ data have been taken from the manufacturer datasheet, so no temperature dependence has been considered. The solar cell used has an efficiency of $40.5 \%$ at $500 \mathrm{X}$.

\section{RESULTS}

FK concentrator has been measured and simulated for five different positions of the receiver (i.e. SOE+cell), maintaining POE at a fixed position. This means that for each situation analyzed, the receiver has been placed at a different point of the red line shown in FIGURE 1. $z=0$ is the nominal position, where POEcell distance is $250 \mathrm{~mm}$ and best module performance is obtained. For positions with $z>0$ the receiver is moved towards the POE. Analogously, for positions with $\mathrm{z}<0$ the receiver is moved away from the POE.

\section{Simulated irradiance profiles}

FIGURE 2 shows the simulated irradiance profiles for the five receiver positions $(z=8,4,0,-4,-8)$, and for three ranges of wavelengths fitting with the EQEs of the three junctions (Top Cell or TC; Middle Cell or $\mathrm{MC}$; and Bottom Cell or $\mathrm{BC}$ ).

Let us analyze the profiles obtained. As expected, for the whole range of receiver positions the FK preserves very uniform profiles for the three junctions, being the nominal position $(z=0)$ the most favorable situation. Besides, the profile uniformity of the TC is better preserved for all the positions than those of $\mathrm{MC}$ and BC. For $z>0$ positions, rays impinge on SOE surface at 


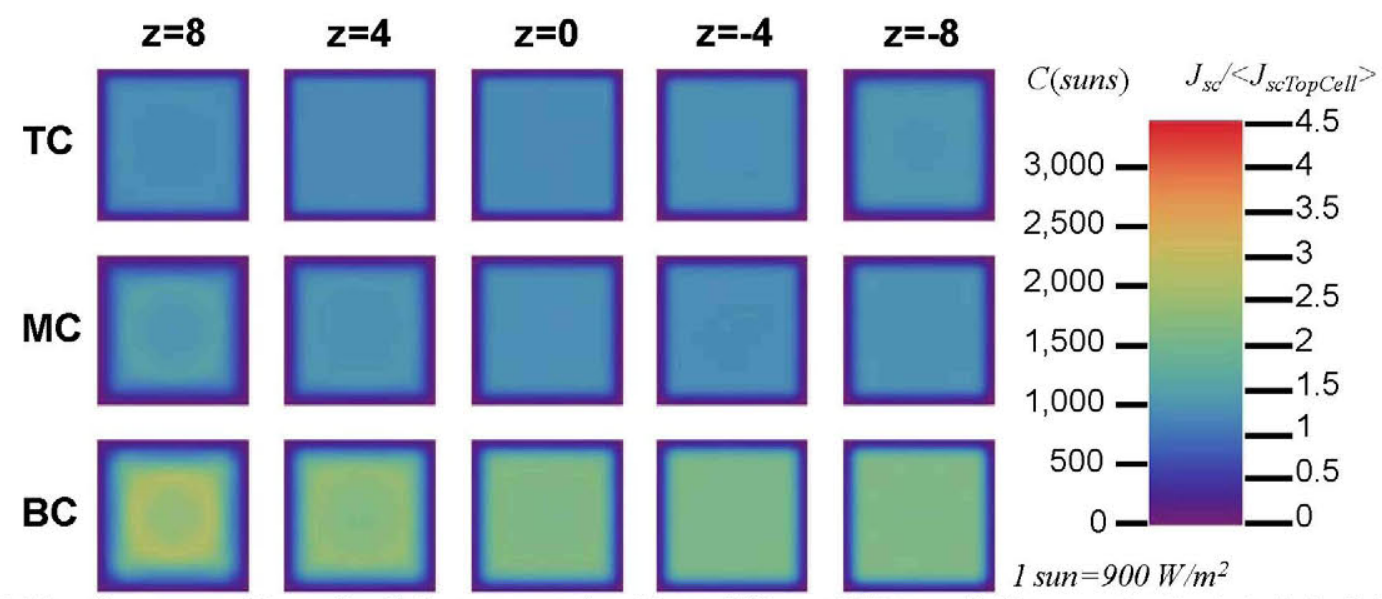

FIGURE 2 Irradiance maps/Normalized photocurrent densities at different POE-to-cell distances $(\mathrm{z}=-8,-4,0,4,8$, all in $\mathrm{mm})$ for the top cell (TC), middle cell (MC) and bottom cell (BC).

a low position, producing non-uniformities (especially remarkable for $z=8$ in the $\mathrm{MC}$ and $\mathrm{BC}$ ) and efficiency losses appear as a consequence. On the other hand, a different situation appears for $\mathrm{z}<0$ positions: rays suffer cross-talk on the SOE surface and some of them are lost (especially rays corresponding to TC in $z=-8$ situation, where dark frame around the light spot is thicker, due to light losses).

\section{I-V curve comparison}

This subsection analyzes the I-V curves measured outdoors with the manufactured FK prototype and those simulated with our model, for the five different situations aforementioned. FIGURE 3 shows the different I-V curves, each one of the five graphics containing a simulated curve and its corresponding measured one, and a table with $I_{s c}, F F$ and efficiency values for each curve. Certain mismatch in absolute values can be observed when comparing measured and simulated curves. This is due to some degree of uncertainty introduced in the model through: an underestimation of the series resistance in the cell; a difference in solar cell efficiencies under uniform irradiance (the measured cell belongs to the $39.0 \%$ bin while the simulated cell presents a $40.5 \%$ efficiency); MJ cell $J_{s c}$ (a) 1 sun values have been taken directly from manufacturer's datasheet instead of experimentally measured; temperature effects have not been considered in cell and optical models.

FIGURE 4 shows the evolution of $I_{s c}, F F$ and efficiency values when POE-cell distance is modified within the range specified above $(-8 \mathrm{~mm}<z<8 \mathrm{~mm})$. It can be concluded that dependences of the three parameters vs. $z$ follow similar trends in measurement and simulation results, validating in this way the cell model behavior regarding non-uniformities and dispersion. Secondly, FF (and consequently efficiency) in both measurements and simulations, is more sensitive to chromatic dispersion (dominating effect in the $z>0$ region, as explained above) than to light loss (appearing in $z<0$ for TC), since efficiency losses are higher for $z>0$ region. Best results are obviously obtained for the situation with the lowest dispersion (i.e. nominal position). Finally, $F F$ and efficiency variations with $z$ are slightly larger for simulated than for measurement results, implying that simulations are more sensitive to non-uniformities and chromatic dispersion than measurements.

\section{CONCLUSIONS}

This work has confirmed CPV systems measurement and simulation results dependences with POE-cell distance, through the FK concentrator particular case. Results point out that uniform irradiance and low chromatic dispersion are vital issues in order to obtain high FF and efficiency, so a concentrator able to provide even irradiances over wide ranges of receiver positions (i.e. system misalignments) is compulsory to succeed in CPV. FK has demonstrated its insensitivity to chromatic aberration in module misalignment situations, maintaining its excellent uniformity on the cell. Furthermore, FK can obtain significantly better results for lower $J_{\text {ratio }}(\sim 1)$ values with published measured efficiencies over $32 \%$ [4].

\section{ACKNOWLEDGMENTS}

Authors thank the European Commission (SMETHODS: FP7-ICT-2009-7 Grant Agreement No. 288526, NGCPV: FP7-ENERGY.2011.1.1 Grant Agreement No. 283798, METACELLS: Marie Curie program), the Spanish Ministery of Economy and Competitiveness
(ENGINEERING 
METAMATERIALS: CSD2008-00066, SEM: TSI020302-2010-65 SUPERRESOLUCION: TEC201124019, TEC2011-28639-C02-01, SIGMAMODULOS: IPT-2011-1441-920000, PMEL: IPT-2011-1212920000), the Comunidad de Madrid under the NUMANCIA II program (S2009/ENE1477 and UPM (Q090935C59) for the support given to the groups research activity, making the present work possible.
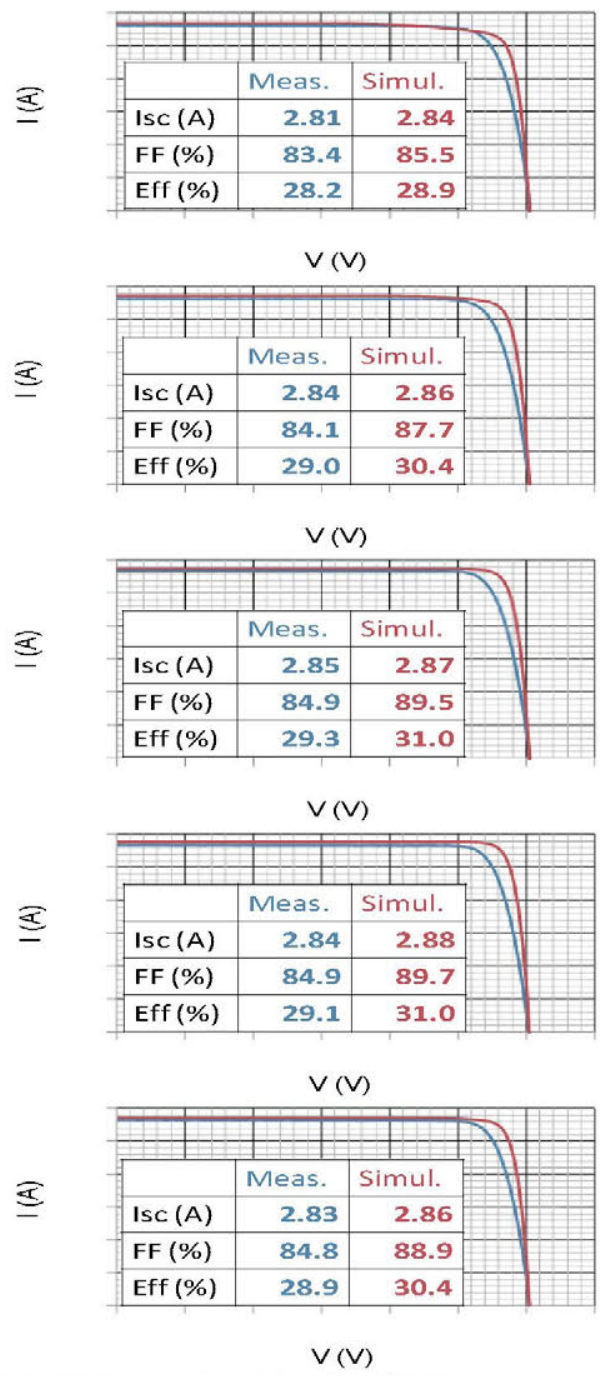

FIGURE 3 Measured and simulated I-V curves. From top to bottom: $\mathrm{z}=8, \mathrm{z}=4, \mathrm{z}=0, \mathrm{z}=-4$ and $\mathrm{z}=-8$ (all in $\mathrm{mm}$ ).

\section{REFERENCES}

1. P. Benítez et. al. "High performance Fresnel-based photovoltaic concentrator", Optics Express, Vol. 18, Issue S1, pp.A25-A40, april 2010.

2. P. Espinet, et. al. "Extended description of tunnel junctions for distributed modelling of concentrator multijunction solar cells" Solar Energy Materials and Solar Cells, 2011, vol. 95 n9, pp. 2693-2697.
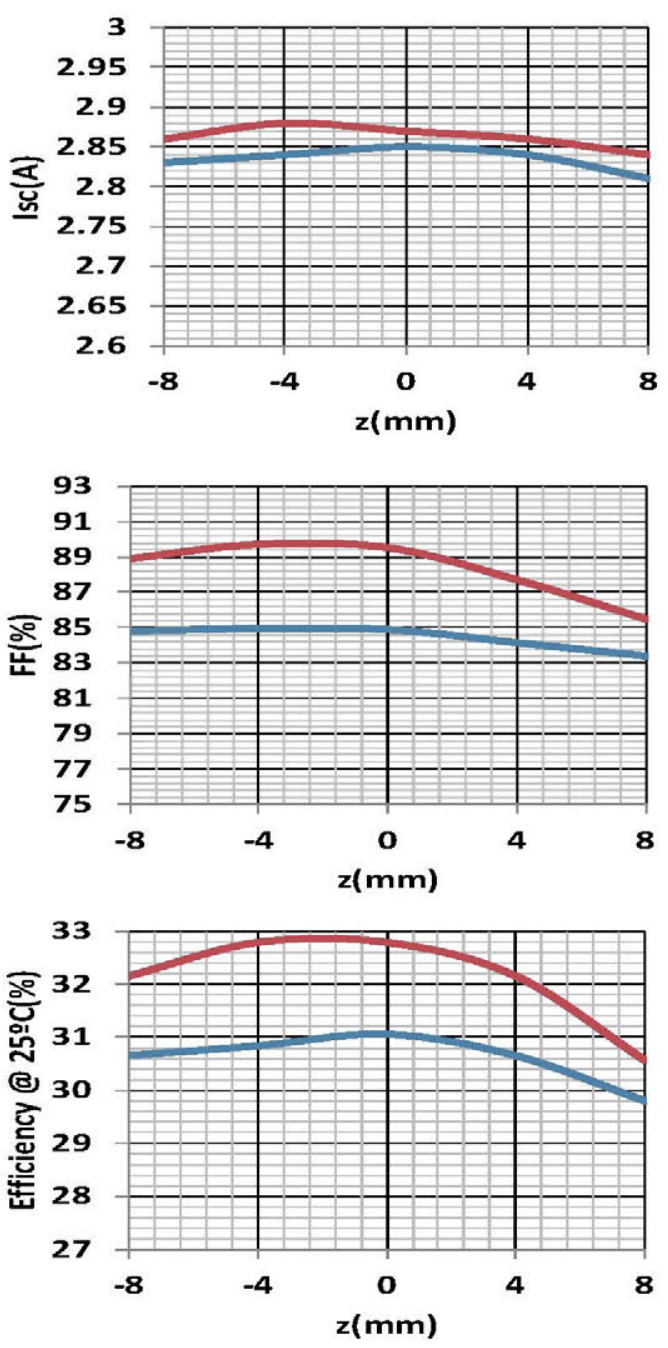

FIGURE $4 I_{s c}, F F$ and efficiency dependence with $z$ position and comparison between simulations (red) and measurements (blue).

3. P. Espinet, et. al. "Triple-junction solar cell performance under Fresnel-based concentrators taking into account chromatic aberration and off-axis operation" Proceedings of the $8^{\text {th }}$ International Conference on Concentrating Photovoltaic Systems, April 2011, Toledo (Spain).

4. P. Zamora, et. al. "Experimental characterization of Fresnel-Köhler concentrators," J. Photon. Energy. 2(1), 021806 (Oct 16, 2012).

5. I. García et. al. "Extended triple-junction solar cell 3D distributed model: application to chromatic aberration related losses", Proceedings of the $7^{\text {th }}$ International Conference on Concentrating Photovoltaic Systems, April 2011, Las Vegas, Nevada, (USA). 